Christian Mélot

\title{
To score or not to score during triage in the emergency department?
}

Received: 6 April 2015

Accepted: 7 April 2015

Published online: 14 May 2015

(C) Springer-Verlag Berlin Heidelberg and ESICM 2015

\section{Mélot (『)}

Head of the Emergency Medicine Department, Erasme University Hospital, Lennik Road 808, 1070 Brussels, Belgium

e-mail: cmelot@ulb.ac.be

Tel.: +32-2-555.33.82

In this issue, Coslovsky et al. [1] report a model to detect high-risk patients during the triage process in the emergency department (ED) with a very high discriminant power.

Triage is a central task in an ED. In this context, triage is viewed as the rating of patients' clinical urgency. Rating is necessary to identify the order in which patients should be given care in an overcrowded ED. ED triage is a relatively modern phenomenon, introduced in the 1950s in the USA [2].

Since the early 1990s, several countries have developed and introduced ED triage. Most of the triage scales have been designed as five-level scales. Of these, the Australian Triage Scale (ATS), Canadian Emergency Department Triage and Acuity Scale (CTAS), the Manchester Triage Scale (MTS), the French scale (FRenh Emergency Nurses Classification in Hospital), and Emergency Severity Index (ESI) have had the greatest influence on modern ED triage [3-7]. The triage of patients at the ED is crucial to the provision of safe patient care. Accurately triaging patients is difficult, requires experience, and may be subject to inter-rater variability [8].
On the other hand, the Early Warning Scoring System (EWSS), first introduced in 1997 at the James Paget University Hospital in Great Yarmouth (UK), was developed to detect deteriorating physiological parameters in ward patients [9]. More recently, the National Early Warning Score (NEWS), developed in conjunction with the Royal College of Physicians of London, has been more rigorously tested and performs better than any of the 33 published systems commonly in use [10]. It has a good ability to discriminate at risk of cardiac arrest, death, or unexpected intensive care unit (ICU) admission and it is currently being promoted as a standardized system across the UK. The area under the ROC curve was 0.873 for any outcome occurring within $24 \mathrm{~h}$ (cardiac arrest, unanticipated ICU admission, and death) [10]. The NEWS was studied in a Dutch ED and was measured at different time points. It was a good predictor of patient outcomes and can be of additional value in the ED to longitudinally monitor patients throughout their stay in the ED [11].

As in the Coslovsky et al. study, when dealing with a model in medicine two points of view can be statistically evaluated. First, a frequentist approach dealing with a significant $p$ value for the odds ratio (OR) indicating that a factor is associated with the outcome (i.e., death of a patient). This approach gives information on a cohort of patients among which certain will die. Second, a Bayesian approach dealing with a significant discriminant power of a model with a significant ROC curve tending to the ideal test with $100 \%$ of sensitivity and specificity. In the latter, an individual patient can be more clearly discriminated as being at risk of death or not. The relationship between both approaches was discussed in the paper by Pepe et al. [12] and is summarized in Fig. 1. When the OR is very high the discriminant power of the model is high with an area under the ROC curve close to 1 . When we look to the individual ORs retained in the model in the paper by Coslovsky et al. [1], we see ORs moderately deviating from 1 (no effect) except for low Glasgow Coma Scores 


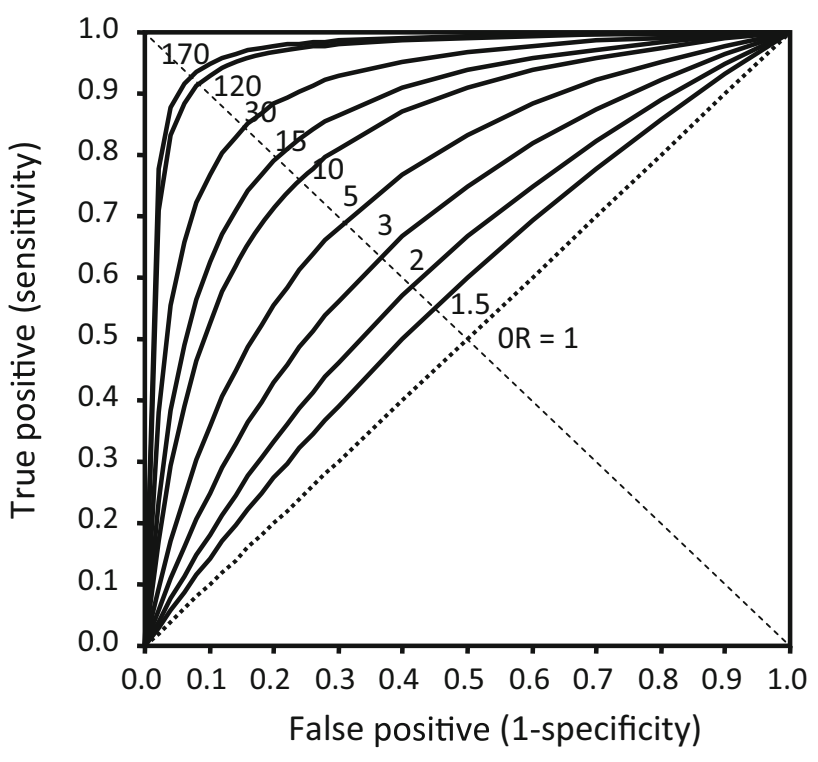

Fig. 1 Relationship between odds ratio (OR) given by logistic regression (frequentist approach) and the ROC curve (Bayesian approach). Modified from ref. [12]

with ORs above 20. But, when we pull all the significant ORs together we reach a highly discriminant multivariable model with an area under the ROC curve of 0.922 (i.e., close to 1), indicating that the final model seems good for selecting patients at risk of death. This area under the ROC curve was among the highest values reported in the literature concerning triage scales in ED.

In his collective book, Daniel Kahneman, the recipient of the Nobel Prize for Economics in 2002, shows that rare causes of death were overestimated and more frequent causes of death were underestimated (Fig. 2) [13]. Thus, it is not surprising in Fig. 3

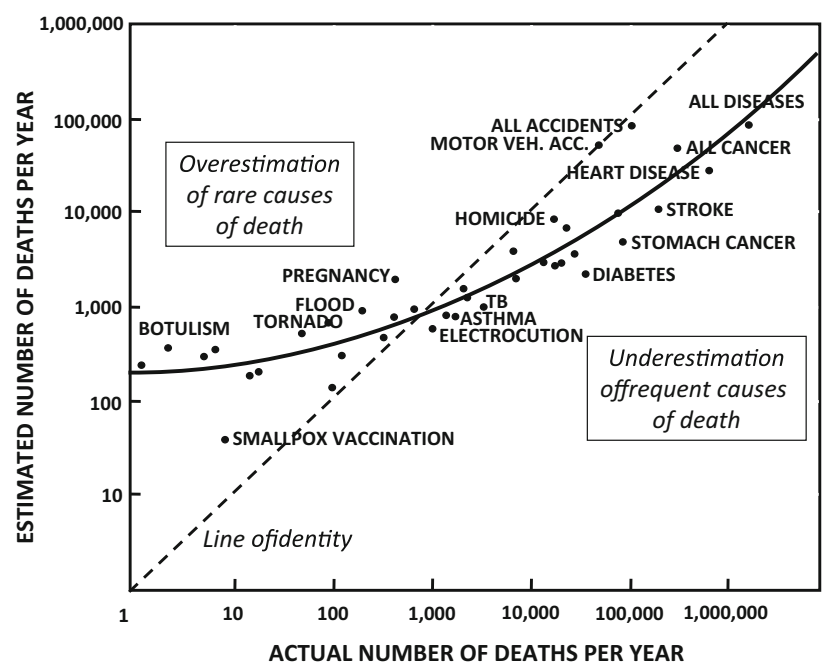

Fig. 2 Relationship between the estimated and the actual annual risk of deaths. Modified from ref. [13]

of the paper by Coslovsky et al. [1] that the nonsystematic subjective nurses' evaluation is higher for the low risk of death and becomes lower for the very high death rate, indicating that the subjective evaluation is biased.

In conclusion, the paper by Coslovsky et al. [1] shows that a structured evaluation of the triage process in an ED in a single center is better for predicting patients at risk of death and therefore requiring rapidly taking in charge. This multivariable model should now be validated in a multicenter study before being widely used.

\section{Conflicts of interest None.}

\section{References}

1. Coslovsky M, Takala J, Exadaktylos AK, Martinolli L, Merrz TM (2015) A clinical prediction model to identify patients at high risk of death in the emergency department. Intensive Care Med. doi:10.1007/s00134-015-3737-x

2. Gilboy N, Travers D, Wuerz R (1999) Re-evaluating triage in the new millennium: a comprehensive look at the need for standardization and quality. J Emerg Nurs 25:468-473

3. Australasian College for Emergency Medicine's (ACEM), policy and guideline on triage and the College of Emergency Nursing Australasia (CENA) (2013) position statements on triage.

http://www0.health.nsw.gov.au/ policies/pd/2013/pdf/PD2013_047.pdf. Accessed 30 Mar 2015
4. Murray M, Bullard M, Grafstein E (2004) Revisions to the Canadian emergency department triage and acuity scale implementation guidelines. CJEM 2004(6):421-427

5. Parenti N, Reggiani ML, Iannone $P$, Percudani D, Dowding D (2014) A systematic review on the validity and reliability of an emergency department triage scale, the Manchester triage system. Int J Nurs Stud 51:1062-1069. doi:10.1016/j.ijnurstu.2014.01.013

6. Taboulet P, Moreira V, Hass L, Porcher $\mathrm{R}$, Braganca A, Fontaine JP, Poncet MC (2009) Triage with the French emergency nurses classification in hospital scale: reliability and validity. Eur J Emerg Med 16:61-67
7. Eitel DR, Travers DA, Rosenau AM, Gilboy N, Wuerz RC (2003) The emergency severity index triage algorithm version 2 is reliable and valid. Acad Emerg Med 10:1070-1080

8. Farrohknia N, Castrén M, Ehrenberg A, Lind L, Oredsson S, Jonsson H, Kjell Asplund, Göransson K (2011) Emergency department triage scales and their components: a systematic review of the scientific evidence. Scand J Trauma Resusc Emerg Med 19:42

9. Wright MM, Stenhouse CW, Morgan RJM (2000) Early detection of patients at risk (PART). Anesthesia 55:391-392 
10. Smith GB, Prytherch DR, Meredith P, Schmidt PE, Featherstone PI (2013)

The ability of the national early warning score (NEWS) to discriminate patients at risk of early cardiac arrest, unanticipated intensive care unit admission, and death. Resuscitation 84:465-470

11. Alam N, Vegting IL, Houben E, van Berkel B, Vaughan L, Kramer MHH, Nanayakkara PWB (2015) Exploring the performance of the national early warning score (NEWS) in a European emergency department. Resuscitation 90:111-115
12. Pepe MS, Janes H, Longton G, Leisenring W, Newcomb P (2004) Limitations of the odds ratio in gauging the performance of a diagnostic, prognostic, or screening marker. Am J Epidemiol 159:882-890
13. Slovic P, Fischhoff B, Lichtenstein $\mathrm{S}$ (2008) Facts versus fears: understanding perceived risk. In: Khaneman D, Slovic P, Tversky A (eds) Judgment under uncertainty: heuristics and bias, 24th edn. Cambridge University Press, New York, pp 463-489 